Review Article

\title{
Science Today - Lessons Learnt and Suggestions for the Youngsters
}

\section{PK Rajagopalan}

Former Director, Vector Control Research Center, Indian Council of Medical Research and formerly: WHO STAC Member, WHO Consultant and WHO Expert Committee Member on Malaria, Filariasis and Vector Control.

DOI: https://doi.org/10.24321/0019.5138.202032

\section{I $\quad \mathbf{N} \quad \mathbf{F} \quad \mathbf{O}$}

\section{E-mail Id:}

leelaraj2004@gmail.com

Orcid Id:

https://orcid.org/0000-0002-8324-3096

How to cite this article:

Rajagopalan PK. Science Today - Lessons Learnt and Suggestions for the Youngsters.

J Commun Dis 2020; 52(3): 58-61.

Date of Submission: 2020-03-12

Date of Acceptance: $2020-04-26$

\section{$\begin{array}{llllllll}\mathbf{A} & \mathbf{B} & \mathbf{S} & \mathbf{T} & \mathbf{R} & \mathbf{A} & \mathbf{C} & \mathbf{T}\end{array}$}

As a 90-year-old vintage scientist, who started his career in 1952, I felt extremely honoured to attend the annual meeting of Indian Society of Malaria and Other Mosquito borne diseases (ISMOCD) at Kochi, Nov 29 - Dec 1, 2019. It was one of the best organized meetings, inaugurated by India's Health Minister, and well attended by both young and old. The scientific deliberations were excellent. But what will an old man like me speak at the meeting. But when I saw hundreds of young and aspiring scientists at the meeting (some of them told me that they are attending to meet other scientists of repute and get inspired), I felt extremely happy and proud. I thought this will be the ideal forum, to present my views about the deficiencies in our approach to research on solving health problems at present. Our future hope lies in these youngsters. I am grateful to the dynamic organizer of the meeting, for providing this opportunity.

In the 1930s, Hitler's Germany and Stalin's Russia prescribed "thrust areas in research - damaging science to such an extent that many scientists migrated to other countries. Our present-day situation is also similar these days. The priority areas in biomedical research now in India get funding only if there are catchy phrases Molecular Biology, Climate change etc. Foreign Collaboration has now reached such a ridiculous extent that we have become suppliers of raw material for research (e.g. human blood samples infected with malaria/ filarial parasites) to foreign collaborators for their research work, in return for dollar grants, foreign trips, etc. Such foreign collaboration has stifled indigenous meaningful research, and every scientific worker- especially those who return to India after higher training abroad- wants now an air conditioned laboratory, costly imported equipment's and want to publish papers which may not have any relevance in solving public health problems in India. The problems are in the field, as far as public health is concerned, and emphasis should have been on working in the field. Unfortunately, it is a fundamental problem in our polity - as in many parts of the world - science and research are no longer directed towards problem solving. Biomedical research is oriented towards palliatives, that continues to rake in those who finance research, not in curing. According to Dr. Mahler, the then world Health Organization (WHO) DG. He felt that indigenous planning and working did not suit 
those who were "Market fundamentalists and free trade theologians".

The question is how much of the research work done in India is relevant to the health needs of the country. The government has been very liberal with funds. Over the years, many research institutions have been created in different parts of India to deal with different diseases and in different regions. Some of them are doing almost nothing to contribute to the immediate health needs of the country; and some just replicating the work of other institutions. But all of them publish a number of papers in Journals. There are also a few good institutions headed by brilliant and committed scientists. But their impact is masked by others. In practical terms, the fundamental question of relevance of present-day research to country's immediate health needs (as Smt. Indira Gandhi pointed out in the 1980s) still remains unanswered.

I may be permitted to write my views on a few diseases and topics, (list not exhaustive) hoping they merit the attention of numerous youngsters here.

Firstly, our youngsters should understand some basics. They must study while working in the field. The textbooks and Universities do not provide these. In nature there exists a complex host-parasite relationship among various animals, their arthropod vectors and infective organisms. While the role of animals, vectors and man in the natural cycle of disease transmission was established about two centuries ago, not much importance has been given to understanding the role of the environment and the necessity of an ecological approach to study this. Pavlovsky's theory of "natural nidality" gave us a concept that wild enzootic foci of many diseases exist in nature independently of man and domestic animals. These foci present well-defined ecological peculiarities wherein pathogens and natural hosts are associated, often through intermediate vector. Many environmental factors like climate, soil, vegetation and topographical features also influence an epidemic. These serve as reliable indicators of the appearance of certain diseases. These natural foci, which may be called "silent zones of diseases", will remain undetected until susceptible human beings come into contact with them. This is exactly what happened, when KFD (Kyasanur Forest Disease) was detected in Shimoga Dt of Karnataka.in 1957, it was purely accidental. The discovery of KFD and the intense ecological and epidemiological studies for nearly 15 years two decades were the result. The rest is all history. The excellent work was summarized by Jorge Boshell, which is like Bible for all scientists. I was lucky to be part of the team for all those years, and mentored by stalwarts. We have now lost the initiative and interest to study arboviruses since the Rockefeller Foundation left and interest is now on molecular studies on viruses, virus containment. Intense Field studies has been downgraded. KFD has now become endemic in many pockets all along the Western Ghats of India and there have been have been sporadic outbreaks including human deaths in different pockets all along the entire Western Ghats. KFD is a tick transmitted viral disease. Present-day investigators do not seem to understand what to look for when they visit new areas from where KFD is being reported. They collect ticks and dead monkey tissues and isolate virus, and publish a paper. KFD is a zoonotic disease. The virus exists in an enzootic cycle with the involvement of several species of small mammals and passerine birds which inhabit the forests adjoining human habitations. These avian and mammalian hosts also circulate high titers of KFD virus for various durations, infecting their tick ectoparasites, have a quick population turnover and therefore play an important role in the natural cycle. They also distribute infected ticks within the range of their movement. New susceptible hosts replace the immune ones in quicker time. The most important small mammal is the common shrew, Suncus murinus an insectivore which is heavily - disproportionate to its size - parasitized simultaneously by both Haemaphysalis (the vector to man and monkeys) and Ixodes sp. It was found that the small mammals, particularly the shrews, the transfer of infection to Ixodes spp. from Haemaphysalis ticks because of simultaneous infestation. This is very important in the epidemiology of KFD virus, since the population of Ixodes predominates that of Haemaphysalis during the prolonged monsoon season, when both human cases and monkey deaths are rare, and helps in the trans - monsoonal survival of the virus. My studies had shown that tick burdens on rodents and shrews are affected by a complex combination of local climate and host factors, making some individuals more likely to contribute to the enzootic transmission cycle. To better understand tickborne transmission, one must study intrinsic factors which have major impact on the small mammals' contribution to the enzootic transmission. Ixodes spp. is the medium of survival of the KFD virus during the monsoon season, since they survive for longer duration during the heavy rains. The nymphs of Haemaphysalis turturis have also been found to help in the trans-monsoonal survival of the virus. Then there is the question of the soft tick, Ornithodorus, from which KFD virus has been isolated, and its host, the insectivorous bat, Rhinolophus rouxii, from which also virus has been isolated. Ornithodorus can also be considered a reservoir, as this tick can stay alive for several years, and attack hosts like bats. The KFD ecosystem study is like a University giving knowledge, and our youngsters should graduate from it.

What about Japanese Encephalitis. There is no man to man transmission through mosquitoes. After getting an infected blood meal, the mosquito must wait till its egglaying is complete - which takes 7-10 days - before it can 
bite another man. These are fundamental aspects. It is because of the massive numbers of mosquitoes produced following monsoon/ heavy rainfall; transmission takes place at all! The time to launch vector control measures is when the conditions are ripe for vectors to start biting humans, not after detecting the first case. In a country like India, where drought and floods occur periodically or with regular frequency, the occurrence of JE can be forecast. Large water pools ideal for breeding of vectors of JE are conducive for nesting and breeding of Ardeid birds such as egrets and herons. The mosquitoes are there, the avian hosts are there and in the adjacent villages' cattle, poultry and pigs are present. JE will strike humans when all these factors co-exist. One can therefore predict with reasonable accuracy an impending epidemic. In fact, research on methods of predicting an epidemic should be a continuous process. Instead the authorities prefer to carry out firefighting operations almost when the epidemic is waning. There does not seem to be a long term plan for JE control. The government's efforts to produce an effective vaccine are laudable. But having a three-dose vaccine is difficult to administer on a mass scale. Who is the target population to be vaccinated? To prevent recurrence of JE epidemics, the first priority is to delimit endemic areas where JE has occurred in the past. The meteorological, animal husbandry, agricultural and revenue departments should coordinate their efforts to share data to predict any unusual changes in ecology and ecosystem. Once it is possible to predict an epidemic, effective use of insecticidal residual sprays will help slow down transmission, provided this is done before the epidemic starts. Strengthening the infrastructure to recognize cases immediately and transport them to the nearest hospital is the only way to save lives (as I did with great success in West Bengal in 1976). JE cases will still occur but the intensity of the epidemic will be low and manageable. At least deaths can be prevented. The missing knowledge is how and from where does the JE virus get introduced into an area where the epidemic occurs? What happens to the virus during the inter-epidemic period? Even if the grey herons and some Ardeid birds are infected, how is the infection transferred to the fledglings? Are there other animals or birds involved in the natural cycle? Is it a zoonotic disease with an animal reservoir for JE virus?

A lot of ignorance is there about the sylvatic cycle of dengue/ Chikungunya complex. In presence of Aedes aegypti, dengue always occurred in endemic form. It was suggested as far back as 1931 that there might be a sylvatic cycle with Aedes albopictus as vector and with monkeys as sylvatic hosts. A strain of this virus was isolated from Aedes africanus caught in Zika forest indicating that there is also a sylvan cycle other than man/ Aedes aegypti/ Aedes albopictus cycle. The haemorrhagic dengue and Chikungunya disease are essentially urban diseases with a cycle between man and mosquito but there may be sylvatic cycles of these viruses in endemic phase. Is there an enzootic cycle involving small rodents and shrews which have large population turnovers and can be ideal participants in the natural cycle? The possibility of non-mosquito vectors like Gamasid and Laelaptid mites to maintain a rodent - mite - rodent chain also should be investigated. Lot more studies are needed to establish this.

Another important neglected disease (not a new one to science) is scrub typhus and many cases have been reported from various parts of India in recent months. The late Dr J. R. Audy (The founder Director of the Institute of Medical Research in Kuala Lumpur) had spent almost a life time working on this disease - with a fascinating epidemiology. The vectors are Trombiculid mites. The adults are $1 \mathrm{~mm}$ long, the hexapod larvae are parasites on animals for a few days only and then return to soil and moult twice. The octopod adults are nonparasitic free-living forms but predating on soil arthropods. The whole life cycle is completed in 2-3 months. Engorged larvae detach from the host in batches, so that a small patch of soil becomes "seeded" with trombiculids. This is the "mite colony" of Audy. Such a colony will perish unless a proportion of larvae return to the same place after second engorgement on the "maintaining host", usually field rats, mice etc. Other incidentals hosts are birds such as warblers, pheasants, quails, fowls etc. These are important as they distribute the mites over a longer range. Man is only a casual host and is of no importance in the life cycle of the vector. Vectors of Rickettsia tsutsugamushi infection in man are caused by several species of Leptotrombidium (formerly Trombicula), always restricted to small ground living mammals. According to Audy, the geographical range of scrub typhus contains the centre of evolution of the mite and of the small rodent hosts. Ecological characteristic of the vector habitat is of extraordinary interest. The infested site which usually comprises several mite colonies and is called a "mite island". The "mite colony" is usually seeded from a single warmblooded host, while the "mite island "may be maintained from several hosts of the same or different species. Where suitable population of hosts is present in patches but "mite islands "are absent, the site is called a "receptive focus". The vector completes its life cycle in the soil if it has the required moisture contents. When the soil becomes dry, the larvae disappear, while the adults tend to disperse more widely and stop laying eggs. This explains the relationship of the epidemiological units of infection, called "Typhus islands" (by Audy), to vegetation and ground features. A "Typhus island" is a mite island infected by $R$. tsutsugamushi from either an infected mite or a host from the neighboring "typhus island". Transfer of Rickettsia from rodent host to uninfected larvae maintains the epidemiological chain. It is likely in the course of successive passages that the 
virulence of infective agent increases. The Trombiculid mites are generally found feeding together in clusters in the ears of rats and this facilitate mite are generally found feeding together in clusters in the ears of rat and this facilitate the transfer of the infective agent. Rickettsia can be transmitted transovarially from adult mites to larvae. Isn't this a fascinating story? How many of our experts heading various departments, or holding chairs of eminence, or who talk in conferences displaying colorful slides really know these. One has to spend long time in the field to understand these.

What about Zika virus, which has created a stir (or fear) in both the new and old world. We have known Zika for 70 years, but it was never considered a public health problem. Zika was isolated for the first time in Uganda in 1947 from a sentinel monkey and later from Aedes africanus mosquitoes. There are also reports of isolations from Ae. aegypti. It's presence in India was noted in 1950s (Smithburn, Kerr and Gatne, 1954. Available from: Smithburn, Kerr and Gatne, 1954) when significant numbers of the human sera collected neutralized Zika. There was no isolation of Zika virus from the Indian subcontinent. Zika virus, along with Ebola are potential dangers to our country in the not too distant future. And Zika and dengue have close similarities with Yellow fever and about which no studies have been made in India. At least two factors contribute to re-emergence of several viral diseases; rapid human population growth near tropical forests and the fact that some viruses like sylvatic dengue has been shown to adapt to human hosts and urban mosquitoes. Dengue has also become acute in areas bordering forests, e.g. Kottayam in Kerala. There is little or no adaptive barrier to the emergence of sylvatic dengue into human population. What about interepidemic cycles of dengue and Chikungunya viruses and the possibility of a zoonotic cycle. We should carry out long term multidisciplinary investigations in the field, particularly in the deep jungles of India and their interfaces.

Finally, I must talk a little about global warming and climate change. A lot of unnecessary scare is being created. There are lots of references in the literature. There was a good article in the Annals of New York Academy of sciences. The simplest connections are through temperature, affecting the biting, survival and reproductive rates of the vectors, and the survival and development rates of the pathogens that they carry. Precipitation also exerts a very strong influence, most obviously in the case of diseases transmitted by vectors that have aquatic developmental stages (such as mosquitoes), but also, via humidity, on diseases transmitted by vectors without such stages, such as ticks or sand flies. Climate and weather conditions also exert a range of more indirect effects, through wider effects on the natural environment and on human systems, for example as drought may affect water-storage, land-use and irrigation practices, and population movement, in turn, affecting vector ecology, and human exposure to infection. For example, a recent WHO report summarizing the importance of vector-borne diseases states that previously relatively stable geographical distributions are now changing owing to a range of factors, 'including climate change, intensive farming, dams, irrigation, deforestation, population movements, rapid unplanned urbanization, and phenomenal increases in international travel and trade' These views were expressed during the last century itself, by Pavlovski, in his theory of Natural Nidality of Infectious Diseases.

What one wishes for and what really is possible are two different things in India. First of all, the conditions existing now are not the same as in olden days. The attitude of the present generation of scientists and their outlook is different, even though they may be intelligent, educated, and sincere. Many scientists are not prepared to stay for long durations in the field (I spent 17 years in a field station), because their future career, now, depends on the number of "quickies" they publish. There is also a craze for foreign collaboration, because of easy funding. Even the British Medical Council is now in the fray to "investigate" KFD, with their own objectives. Will they solve the problem, or is it another Safari Research?

A lot of introspection is needed and there should be fresh thinking by our aspiring youngsters. 